M. Itô

Nagoya Math. J.

Vol. 57 (1975), 121-125

\title{
DÉMONSTRATION SIMPLE DE LA REPRESENTATION INTÉGRALE DU NOYAU COMPLÈTEMENT SOUS-HARMONIQUE ET INVARIANT PAR ROTATIONS
}

\author{
MASAYUKI ITÔ
}

\section{§ 1. Introduction}

Dans toute la suite $\boldsymbol{R}^{n}$ désigne l'espace euclidien à dimension $n(\geqq 1)$. On désigne par $\Delta$ l'opérateur de Laplace sur $\boldsymbol{R}^{n}$. Dans la théorie du potentiel, un noyau de convolution sur $\boldsymbol{R}^{n}$ signifie une mesure de Radon positive dans $\boldsymbol{R}^{n}$. Rappelons qu'un noyau de convolution de Dirichlet $N$ sur $\boldsymbol{R}^{n}$ est un noyau de convolution sur $\boldsymbol{R}^{n}$ tel que $1 / \hat{N}$ soit égal à une fonction définie-négative dans $\boldsymbol{R}^{n}$ à valeurs réelles, où $\hat{N}$ désigne la transformée de Fourier de $N$ (cf. [1]). Pour un nombre $p>0, G_{p}$ désigne le noyau de convolution de Dirichlet sur $\boldsymbol{R}^{n}$ vérifiant $(\Delta-p) G_{p}=-\varepsilon(\mathrm{au}$ sens des distributions), où $\varepsilon$ est la mesure de Dirac à l'origine. Si $n \geqq 3$, on note $G=G_{0}$ le noyau newtonien avec $\Delta G=-\varepsilon$. Dans l'article précédent [2], on a montré le théorème suivant:

THÉORÈmE. Soit $N$ un noyau de convolution sur $\boldsymbol{R}^{n} \boldsymbol{s}^{\prime}$ annulant à l'infini ${ }^{1)}$ et invariant par rotations. Alors les deux énoncés suivants sont équivalents :

(1) $N$ est complètement sous-harmonique (en dehors de l'origine); c'est-à-dire, pour tout l'entier $m \geqq 0, \Delta^{m} N \geqq 0$ au sens des distributions en dehors de l'origine, où $\Delta^{0} N=N, \Delta^{1}=\Delta$ et $\Delta^{m}=\Delta^{m-1} \Delta(\forall m \geqq 2)$.

(2) $N$ est de la forme

$$
N=c \varepsilon+\int G_{p} d \nu(p),
$$

où $c$ et $\nu$ sont respectivement une constante $\geqq 0$ et une mesure positive sur $\boldsymbol{R}^{+}=\left\{t \in \boldsymbol{R}^{1} ; t \geqq 0\right\}$.

Received April 17, 1974.

1) Cela signifie que, quelle que soit $f$ une fonction finie et continue dans $R^{n}$ à support compact, $\lim _{x \rightarrow \infty} N * f(x)=0$. 
Si un noyau de convolution $N$ sur $R^{n}$ vérifie l'énoncé (2), alors $N$ est un noyau de convolution de Dirichlet sur $\boldsymbol{R}^{n}$ ou bien 0 . Donc le présent théorème joue un rôle essentiel dans la discussion sur le cône convexe divisible (cf. [2] et [3]).

La démonstration du théorème dans [2] est très compliquée. Le but de cette note est de fournir une démonstration simple du présent théorème, en utilisant seulement le théorème de Bernstein.

\section{§ 2. Une démonstration simple du théorème}

Rappelons d'abord le théorème de Bernstein (cf. par exemple, [4]).

LEMME. Soit $\varphi$ une fonction infiniment dérivable dans $(0,+\infty) \grave{a}$ valeurs réelles. Alors les deux énoncés suivants sont équivalents:

(a) $\varphi$ est complètement monotone; c'est-à-dire, pour tout l'entier $m \geqq 0,(-1)^{m}\left(d^{m} / d t^{m}\right) \varphi \geqq 0$ dans $(0,+\infty)$.

(b) $\varphi$ est de la forme

$$
\varphi(t)=\int \exp (-t s) d \nu(s) \quad(\forall t>0),
$$

où $\nu$ est une mesure positive sur $\boldsymbol{R}^{+}$.

Dans ce cas, $\nu$ est uniquement déterminée, d'après l'injectivité de la transformation de Laplace.

Montrons notre théorème. L'implication (2) $\Rightarrow(1)$ résulte immédiatement du fait que, pour tout l'entier $m \geqq 0$ et pour tout $p \geqq 0, \Delta^{m} G_{p}=p^{m} G_{p}$ en dehors de l'origine. Donc on montrera seulement l'implication $(1) \Rightarrow(2)$. Supposons que l'énoncé (1) a lieu; alors il existe uniquement une constante $c \geqq 0$ et une fonction infiniment dérivable $\varphi(t) \geqq 0$ dans $(0,+\infty)$ telles que $\int_{0}^{1} \varphi\left(r^{2}\right) r^{n-1} d r<+\infty$ et $N=c \varepsilon+\varphi\left(|x|^{2}\right) d x$. Ayant, pour tout l'entier $m \geqq 0$,

$$
\Delta^{m} N=\left(4|x|^{2} \frac{d^{2}}{d t^{2}}+2 n \frac{d}{d t}\right)^{m} \varphi\left(|x|^{2}\right) d x
$$

en dehors de l'origine, on a $\left(2 t\left(d^{2} / d t^{2}\right)+n(d / d t)\right)^{m} \varphi(t) \geqq 0$ dans $(0,+\infty)$. On a, pour toute la fonction infiniment dérivable $f$ dans $\boldsymbol{R}^{n}$ à support compact,

$$
\lim _{x \rightarrow \infty} \int f(x-y) d\left(\Delta^{m} N\right)(y)=\lim _{x \rightarrow \infty} \int \Delta^{m} f(x-y) d N(y)=0,
$$


et la fonction $\left(2|x|^{2}\left(d^{2} / d t^{2}\right)+n(d / d t)\right)^{m} \varphi\left(|x|^{2}\right)$ de $x$ dans $\boldsymbol{R}^{n}-\{0\}$ est sousharmonique. Donc on a

$$
\frac{d}{d t}\left(2 t \frac{d^{2}}{d t^{2}}+n \frac{d}{d t}\right)^{m} \varphi(t) \leqq 0 \quad \text { dans } \quad(0,+\infty)
$$

et par suite

$$
\frac{d^{2}}{d t^{2}}\left(2 t \frac{d^{2}}{d t^{2}}+n \frac{d}{d t}\right)^{m} \varphi(t) \geqq 0 \quad \text { dans } \quad(0,+\infty) .
$$

Soit $k$ un entier $\geqq 1$ et supposons que, quel que soit $m$ un entier $\geqq 0$,

$$
\frac{d^{2 k}}{d t^{2 k}}\left(2 t \frac{d^{2}}{d t^{2}}+n \frac{d}{d t}\right)^{m} \varphi(t) \geqq 0 \quad \text { et } \quad \frac{d^{2 k-1}}{d t^{2 k-1}}\left(2 t \frac{d^{2}}{d t^{2}}+n \frac{d}{d t}\right)^{m} \varphi(t) \leqq 0
$$

dans $(0,+\infty)$. On a alors, pour tout l'entier $m \geqq 0$,

$$
\begin{aligned}
0 \geqq & \frac{d^{2 k-1}}{d t^{2 k-1}}\left(2 t \frac{d^{2}}{d t^{2}}+n \frac{d}{d t}\right)^{m+1} \varphi(t)=2 t \frac{d^{2 k+1}}{d t^{2 k+1}}\left(2 t \frac{d^{2}}{d t^{2}}+n \frac{d}{d t}\right)^{m} \varphi(t) \\
& +(n+4 k-2) \frac{d^{2 k}}{d t^{2 k}}\left(2 t \frac{d^{2}}{d t^{2}}+n \frac{d}{d t}\right)^{m} \varphi(t)
\end{aligned}
$$

dans $(0,+\infty)$. D'après la présente hypothèse, on a

$$
\frac{d^{2 k+1}}{d t^{2 k+1}}\left(2 t \frac{d^{2}}{d t^{2}}+n \frac{d}{d t}\right)^{m} \varphi(t) \leqq 0
$$

dans $(0,+\infty)$. On a aussi

$$
\begin{aligned}
0 \leqq & \frac{d^{2 k}}{d t^{2 k}}\left(2 t \frac{d^{2}}{d t^{2}}+n \frac{d}{d t}\right)^{m+1} \varphi(t)=2 t \frac{d^{2(k+1)}}{d t^{2(k+1)}}\left(2 t \frac{d^{2}}{d t^{2}}+n \frac{d}{d t}\right)^{m} \varphi(t) \\
& +(4 k+n) \frac{d^{2 k+1}}{d t^{2 k+1}}\left(2 t \frac{d^{2}}{d t^{2}}+n \frac{d}{d t}\right)^{m} \varphi(t)
\end{aligned}
$$

dans $(0,+\infty)$. D’après l'inégalité obtenue ci-dessus, on a

$$
\frac{d^{2(k+1)}}{d t^{2(k+1)}}\left(2 t \frac{d^{2}}{d t^{2}}+n \frac{d}{d t}\right)^{m} \varphi(t) \geqq 0
$$

dans $(0,+\infty)$. On obtient ainsi, par récurrence, que pour tous les entiers $k \geqq 0$ et $m \geqq 0$,

$$
(-1)^{k} \frac{d^{k}}{d t^{k}}\left(2 t \frac{d^{2}}{d t^{2}}+n \frac{d}{d t}\right)^{m} \varphi(t) \geqq 0
$$


dans $(0,+\infty)$. D'après le lemme, pour tout l'entier $m \geqq 0$, il existe une mesure positive $\nu_{m}^{\prime}$ sur $\boldsymbol{R}^{+}$telle que

$$
\left(2 t \frac{d^{2}}{d t^{2}}+n \frac{d}{d t}\right)^{m} \varphi(t)=\int \exp (-t s) d \nu_{m}^{\prime}(s)
$$

dans $(0,+\infty)$. Ayant $\lim _{t \rightarrow+\infty}\left(2 t\left(d^{2} / d t^{2}\right)+n(d / d t)\right)^{m} \varphi(t)=0$, on a $\nu_{m}^{\prime}(\{0\})=0$. Donc, en utilisant une certaine transformation, il existe une mesure positive $\nu_{m}$ dans $(0,+\infty)$ telle que

$$
\left(2 t \frac{d^{2}}{d t^{2}}+n \frac{d}{d t}\right)^{m} \varphi(t)=\int-\frac{1}{\left(2 \pi s^{1 / 2}\right)^{n}} \exp \left(-\frac{t}{4 s}\right) d \nu_{m}(t)
$$

dans $(0,+\infty)$. Ayant, pour tout l'entier $m \geqq 0$,

$$
\Delta_{(x)}\left(2|x|^{2} \frac{d^{2}}{d t^{2}}+n \frac{d}{d t}\right)^{m} \varphi\left(|x|^{2}\right)=\left(2|x|^{2} \frac{d^{2}}{d t^{2}}+n \frac{d}{d t}\right)^{m+1} \varphi\left(|x|^{2}\right)
$$

et

$$
\Delta_{(x)}\left(\frac{1}{\left(2 \pi t^{1 / 2}\right)^{n}} \exp \left(-\frac{|x|^{2}}{4 t}\right)\right)=\frac{d}{d t}\left(\frac{1}{\left(2 \pi t^{1 / 2}\right)^{n}} \exp \left(-\frac{|x|^{2}}{4 t}\right)\right)
$$

dans $\boldsymbol{R}^{n}-\{0\}$, on obtient, pour tout l'entier $m \geqq 1$ et pour $t>0$,

$$
\int \frac{1}{\left(2 \pi s^{1 / 2}\right)^{n}} \exp \left(-\frac{t}{4 s}\right) d \nu_{m}(s)=\int \frac{d}{d s}\left(\frac{1}{\left(2 \pi s^{1 / 2}\right)^{n}} \exp \left(-\frac{t}{4 s}\right)\right) d \nu_{m-1}(s) .
$$

Donc on a, quels que soient $k$ un entier $\geqq 1$ et $f$ une fonction infiniment dérivable dans $(0,+\infty)$ à support compact,

$$
\begin{aligned}
k \int & \frac{1}{s^{n / 2}} \int_{s}^{\infty} \exp \left(-\frac{k(t-s)}{4 s}\right) f(t) d t d \nu_{m}(s) \\
\quad & =k \int \frac{d}{d s}\left(\frac{1}{s^{n / 2}} \int_{s}^{\infty} \exp \left(-\frac{k(t-s)}{4 s}\right) f(t) d t\right) d \nu_{m-1}(s) .
\end{aligned}
$$

On peut montrer, d'autre part, qu'il existe un entier $k_{0} \geqq 1$ tel que, quels que soient $k$ un entier avec $k \geqq k_{0}$ et un point $s$ de $\left(0, \frac{1}{2} \min _{t \in \operatorname{supp}(f)} t\right)$,

$$
k \int_{s}^{\infty} \exp \left(-\frac{k(t-s)}{4 s}\right)|f(t)| d t \geqq(k+1) \int_{s}^{\infty} \exp \left(-\frac{(k+1)(t-s)}{4 s}\right)|f(t)| d t .
$$

Par conséquent, en faisant $k \rightarrow+\infty$, on obtient

$$
\int s^{1-n / 2} f(s) d \nu_{m}(s)=\int \frac{d}{d s}\left(s^{1-n / 2} f(s)\right) d \nu_{m-1}(s) .
$$


La fonction $f$ étant quelconque, on a $\nu_{m}=-(d / d t) \nu_{m-1}$ au sens des distributions dans $(0,+\infty)$, d'où, pour tout l'entier $m \geqq 0,(-1)^{m}\left(d^{m} / d t^{m}\right) \nu_{0}$ $=\nu_{m}$ au sens des distributions dans $(0,+\infty)$. Donc il existe uniquement une fonction non-négative et infiniment dérivable $\varphi$ dans $(0,+\infty)$ telle que $\nu_{0}=\varphi(t) d t$ dans $(0,+\infty)$ et pour tout l'entier $m \geqq 0,(-1)^{m}\left(d^{m} / d t^{m}\right) \varphi(t)$ $\geqq 0$ dans $(0,+\infty)$. En utilisant encore le théorème de Bernstein, on peut écrire

$$
\varphi(t)=\int \exp (-p t) d \nu(p) \quad(\forall t>0)
$$

où $\nu$ est une mesure positive sur $\boldsymbol{R}^{+}$. Par conséquent, on a

$$
\begin{aligned}
N & =c \varepsilon+\left(\int \frac{1}{\left(2 \pi t^{1 / 2}\right)^{n}} \exp \left(-\frac{|x|^{2}}{4 t}\right) d \nu_{0}(t)\right) d x \\
& =c \varepsilon+\left(\iint_{0}^{\infty} \frac{1}{\left(2 \pi t^{1 / 2}\right)^{n}} \exp \left(-\frac{|x|^{2}}{4 t}\right) \exp (-p t) d t d \nu(p)\right) d x
\end{aligned}
$$

Ayant

$$
G_{p}=\left(\int_{0}^{\infty} \frac{1}{\left(2 \pi t^{1 / 2}\right)^{n}} \exp \left(-\frac{|x|^{2}}{4 t}\right) \exp (-p t) d t\right) d x
$$

on obtient

$$
N=c \varepsilon+\int G_{p} d \nu(p)
$$

La démonstration est ainsi complète.

\section{BIBLIOGRAPHIE}

[1] A. Beurling and J. Deny: Dirichlet spaces, Proc. Nat. Acad. U.S.A., 45 (1959), 208-215.

[2] M. Itô: Sur les cônes convexes de Riesz et les noyaux de convolution complètement sous-harmoniques, Nagoya Math. J., 55 (1974), 111-144.

[ 3 ] — : Sur l'unicité du cône convexe divisible constitué par de noyaux de convolution de Dirichlet, Nagoya Math. J., à paraître.

[ 4 ] D. Widder: The Laplace transform, Princeton Univ. Press, Princeton, 1948.

\section{Université de Nagoya}

